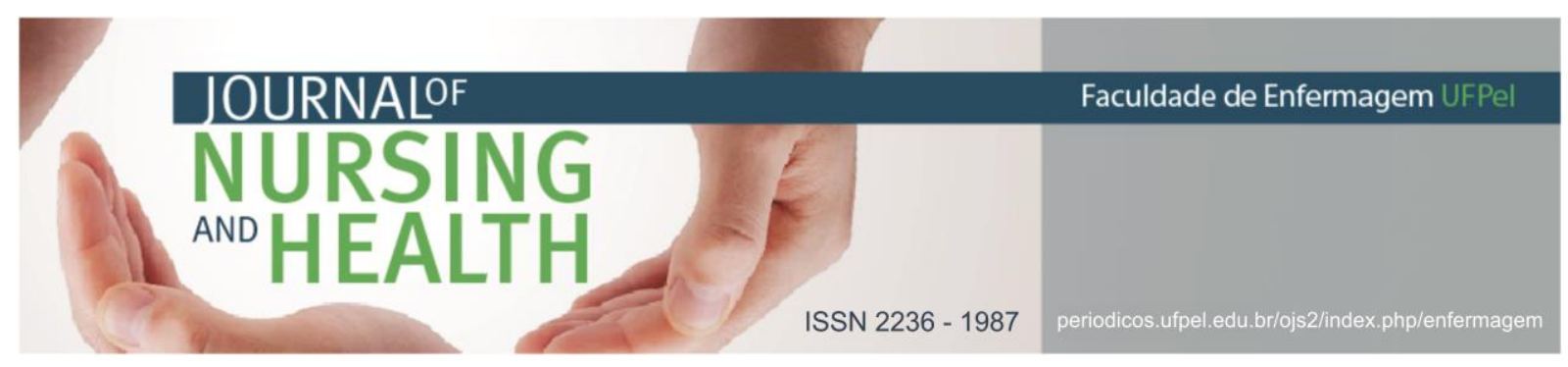

ARTIGO ORIGINAL

\title{
Diabetes gestacional e estado nutricional materno em um hospital universitário de Niterói
}

\author{
Gestational diabetes and maternal nutritional status in a university hospital of \\ Niterói
Diabetes gestacional y estado nutricional materno en un hospital universitário de Niterói

Guerra, Juliana Vidal Vieiraํㅜ Alves, Valdecyr Herdy²; Rodrigues, Diego Pereira³; Branco, Maria Bertilla Lutterbach Riker ${ }^{4}$; Marchiori, Giovanna Rosário Soanno5; dos Santos, Márcia Vieira ${ }^{6}$

\begin{abstract}
RESUMO
Objetivo: identificar o número de casos de diabetes gestacional e correlacionar estado nutricional pré-gestacional e diabetes mellitus gestacional em mulheres atendidas no ambulatório de Ginecologia e Obstetrícia de um Hospital Universitário em Niterói, Brasil. Método: transversal, analítico e retrospectivo. Pesquisa realizada em prontuários de mulheres assistidas durante o parto no ano de 2013, que realizaram no mínimo uma consulta de pré-natal na unidade. As análises estatísticas foram realizadas com o software estatístico Stata 20.0, considerando nível de significância de 5\%. Aprovado pelo Comitê de ética em pesquisa sob parecer 1.354.164/2015. Resultados: $14 \%(n=25)$ desenvolveram diabetes gestacional; 24\% iniciaram a gestação com sobrepeso e 29,5\%, com obesidade. Conclusão: estudos que evidenciam o número de casos do diabetes gestacional a associação a fatores de risco são importantes, principalmente para melhoria da assistência prestada e elaboração de novas estratégias de cuidado.

Descritores: Diabetes gestacional; Cuidado pré-natal; Peso corporal; Índice de massa corporal; Complicações na gravidez.
\end{abstract}

\begin{abstract}
Objective: to identify the number of gestational diabetes cases and to correlate pre-gestational nutritional status and gestational diabetes mellitus in women attended at Gynecology and Obstetrics outpatient clinic of a University Hospital in Niterói, Brazil. Method: transversal, analytical and retrospective. Research carried out in records of women assisted during the birth, in 2013, who performed at least one prenatal visit at the unit. Statistical analyzes were carried out using statistical software Stata 20.0, considering a significance level of 5\%. Approved by the Research Ethics Committee under opinion 1.354.164/2015. Results: 14\% $(n=25)$ developed gestational diabetes; $24 \%$ started with overweight and $29.5 \%$ with obesity. Conclusion: studies that demonstrate
\end{abstract}

\footnotetext{
1 Nutricionista. Mestre em Saúde Materno-Infantil. Universidade Federal Fluminense (UFF). E-mail: julianaguerra.personaldiet@gmail.com http://orcid.org/0000-0002-4509-1343

2 Enfermeiro. Doutor em Enfermagem. Universidade Federal Fluminense (UFF). E-mail: herdyalves@yahoo.com.br http://orcid.org/0000-0001-8671-5063

3 Enfermeiro. Mestre em Enfermagem. Centro Universitário Anhanguera de Niterói (UNIAN). E-mail: diego.pereira.rodrigues@gmail.com http://orcid.org/0000-0001-8383-7663

4 Enfermeira. Mestre em Materno-Infantil. Universidade Federal Fluminense (UFF). E-mail: bertillariker@yahoo.com.br http://orcid.org/0000-0001-6307-4830

${ }^{5}$ Enfermeira. Mestre em Saúde materno-infantil. Faculdade Novo Milênio. E-mail: giovannasoanno@gmail.com http://orcid.org/0000-0002-0675-1679

6 Enfermeira. Mestre em Saúde Materno-Infantil. Universidade Federal Fluminense (UFF). E-mail: enfa.marcia@oi.com.br http://orcid.org/0000-0002-1488-7314
} 


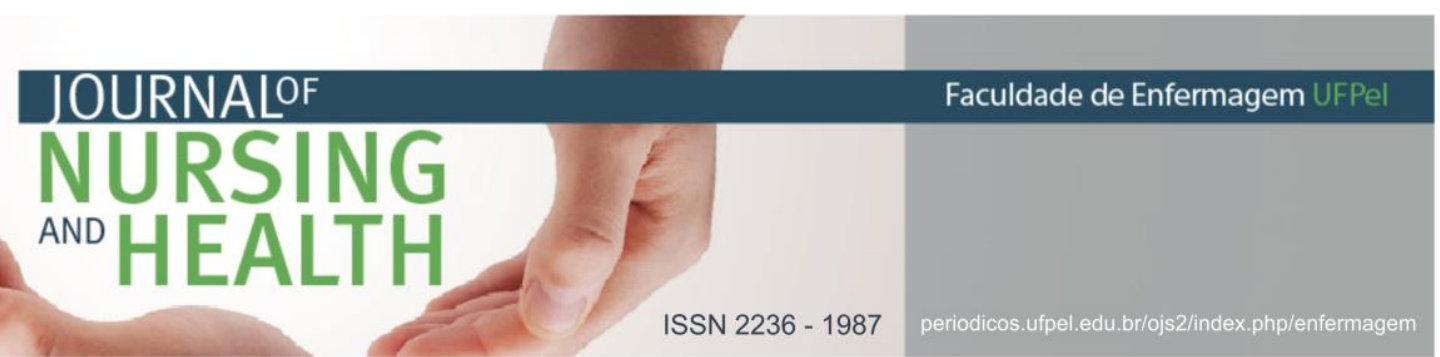

the number of cases of gestational diabetes and the association with risk factors are important, mainly to improve the care provided and to elaborate new care strategies.

Descriptors: Diabetes, gestational; Prenatal care; Body weight; Body mass index; Pregnancy complications.

\section{RESUMEN}

Objetivo: identificar el número de casos de diabetes gestacional y correlacionar estado nutricional pre-gestacional y diabetes mellitus gestacional en mujeres atendidas en el ambulatorio de Ginecología y Obstetricia de un Hospital Universitario en Niterói, Brasil. Método: transversal, analítico y retrospectivo. Investigación realizada en prontuarios de mujeres asistidas durante el parto, en el año 2013, que realizaron como mínimo una consulta de prenatal en la unidad. Los análisis estadísticos se realizaron con el software estadístico Stata 20.0, considerando un nivel de significancia del 5\%. Aprobado en el Comité de ética en investigación por el dictamen 1.354.164/2015. Resultados: $14 \%$ ( $n=25)$ desarrollaron diabetes gestacional; $24 \%$ inició la gestación con sobrepeso y $29,5 \%$, con obesidad. Conclusión: estudios que evidencian el número de casos de la diabetes gestacional la asociación a factores de riesgo son importantes, principalmente para la mejora de la asistencia prestada y la elaboración de nuevas estrategias de cuidado.

Descriptores: Diabetes gestacional; Atención prenatal; Peso corporal; Índice de masa corporal; Complicaciones del embarazo.

\section{INTRODUÇÃO}

O Diabetes Mellitus Gestacional (DMG) é caracterizado por intolerância glicêmica de magnitude variável, sendo detectada pela primeira vez durante a gestação. 0 rastreio do diabetes mellitus gestacional inicia-se logo na primeira consulta do pré-natal com a anamnese clínica aprofundada, quando são observados fatores de risco predisponentes, além da solicitação de exames bioquímicos. ${ }^{1-2}$

A prevalência da DMG é variável, sendo influenciada por fatores geográficos, étnicos e raciais. ${ }^{3}$ Nos Estados Unidos da América, a estimativa é de que $4 \%$ das gestantes sejam acometidas pela doença. Já no Brasil, estima-se que a prevalência esteja entre $2,4 \%$ e $7,2 \%$ das gestantes, sendo a prevalência de obesidade em casos de DMG, de 10,6\%. Considerando que a curto prazo aumentam os riscos para comorbidades na gestante, a longo prazo também amplia o risco para desenvolvimento da síndrome metabólica e do diabetes mellitus tipo 2 na mulher. ${ }^{1,3-4}$

Na América Latina, informações de prevalência de DMG observadas em Córdoba (Argentina) foram de 8,2\%; 8,9\% em Assunção (Paraguai); 5,7\% em La Paz (Bolívia); 7,5\% em Bogotá (Colômbia); 12,7\% na Cidade do México (México) e 7,3\% em São Paulo (Brasil). ${ }^{5}$ A ocorrência do DMG aumenta o risco de complicações clínicas tanto para a mãe quanto para o feto. Estima-se que $50 \%$ das mulheres que desenvolveram o DMG, também serão acometidas pelo diabetes mellitus tipo 2 no prazo de 5 a 10 anos no pós-parto. ${ }^{6}$

Os fatores genéticos, fisiológicos e ambientais relacionados ao desenvolvimento do DMG ainda não foram completamente esclarecidos, mas, evidências sugerem que a hipofunção das células betapancreáticas seja resultante de doença autoimune, ou de causas monogênicas, 


\section{IOURNALIOF \\ NURSING \\ AND HEALTH \\ ISSN 2236 - 1987}

ou ainda de resistência à insulina. ${ }^{7}$ Quanto aos fatores de risco para o desenvolvimento da doença, citam-se: idade (35 anos ou mais), sobrepeso, obesidade ou ganho excessivo de peso na gravidez atual, deposição central exclusiva de gordura corporal, história familiar de diabetes em parentes de primeiro grau, crescimento fetal excessivo, polidrâmnio, hipertensão ou pré-eclâmpsia na gravidez atual, antecedentes obstétricos de abortamento de repetição, malformações, morte fetal ou neonatal, macrossomia, síndrome de ovários policísticos e baixa estatura (menos de 1,5m). ${ }^{8-10}$

O Ministério da Saúde recomenda que as gestantes cumpram o mínimo de seis consultas de pré-natal, nas quais o profissional de saúde deve realizar o acompanhamento e a evolução da gestação, com a avaliação do estado nutricional da mulher prévio à gestação e no momento da consulta utilizando o índice de massa corporal e, também, o índice de massa corporal, segundo a idade gestacional obtidos através de medidas de peso e estatura, e a partir destes, faça a orientação dietética de acordo com as necessidades de cada gestante. ${ }^{11}$ Mulheres que apresentam o estado nutricional inadequado, ou seja, baixo peso ou sobrepeso ou obesidade, estão mais susceptíveis a comorbidades durante a gestação, tais como o diabetes mellitus gestacional e a síndrome hipertensiva específica da gestação ${ }^{12}$, e no caso do diabetes, mulheres que apresentaram a doença durante a gestação.
Considerando o impacto do desenvolvimento do DMG na saúde da mulher e da criança, este estudo objetivou identificar o número de casos de diabetes gestacional e correlacionar o estado nutricional prégestacional e o diabetes mellitus gestacional, em mulheres atendidas no ambulatório de Ginecologia e Obstetrícia de um Hospital Universitário em Niterói, Brasil.

\section{MÉTODO}

Estudo transversal, analítico e retrospectivo, em que se analisou a associação entre o estado nutricional pré-gestacional e 0 diabetes gestacional. Os dados foram coletados nos prontuários de pacientes atendidas na maternidade de um Hospital Universitário em Niterói, Rio de Janeiro, Brasil, referência no atendimento às gestantes de alto risco.

0 projeto de pesquisa foi submetido ao Comitê de Ética em Pesquisa e aprovado sob Parecer n. ${ }^{\circ}$ 1.354.164/2015 (Certificado de Apresentação para Apreciação Ética n. ${ }^{\circ}$ 48561315.8.0000.5243). O período de coleta de dados foi de dezembro de 2015 a março de 2016.

A coleta de dados foi referente ao ano de 2013, e se deu por consulta aos prontuários das gestantes atendidas para parto na maternidade desse Hospital Universitário, sendo o critério de inclusão a realização de, no mínimo, uma consulta de pré-natal no Ambulatório de Obstetrícia e Ginecologia na unidade.

O número de casos de DMG foi observado a partir dos dados de todas 


\section{JOURNALOF \\ NURSING \\ AND HEALTH}

as consultas de pré-natal dos prontuários incluídos no estudo. Para análise descritiva desses dados, as variáveis categóricas foram apresentadas por frequências absolutas e relativas. Em relação às variáveis numéricas, utilizou-se o teste de Qui-Quadrado para apresentá-las. A classificação do índice de massa corporal pré-gestacional foi realizada observando-se a recomendações da Organização Mundial de Saúde para classificação do estado nutricional. ${ }^{13}$ Todos os testes foram apresentados por média e desvio padrão e para a existência de associaçõos entre duas variáveis estatísticas, foi considerado um nível de significância de 5\%. As análises estatísticas foram realizadas com o uso do software SPSS 20.0.

Identificou-se, a partir do livro de Procedimentos Obstétricos da referida Unidade que, no ano estudado, foram atendidas 730 pacientes, das quais 84 $(11,5 \%)$ para a realização de procedimentos obstétricos diversos (aspiração manual intrauterina, curetagem, ou outros procedimentos diferentes de parto) sendo, portanto, seus prontuários desqualificados por não atenderem ao critério de inclusão; $441(60,4 \%)$ prontuários eram de gestantes encaminhadas à Unidade para parto, mas que não foram acompanhadas pelo Ambulatório de Obstetrícia e Ginecologia da mesma durante o pré-natal e, por isso, também foram desconsiderados, e 27 $(3,5 \%)$ prontuários não estavam disponíveis no momento da pesquisa. Assim, somente $178 \quad(24,4 \%)$ prontuários foram selecionados porque atenderam ao critério de inclusão no estudo.
ISSN 2236 - 1987

\section{RESULTADOS}

A análise dos dados referentes aos dados sociodemográficos e histórico de consultas médicas e nutricionais no pré-natal, permitiu identificar que a média de idade das participantes era de 27,7 anos (D.P.= 7,2 anos). Já com relação às consultas do pré-natal, a média foi de 1,9 consultas com a nutricionista (D.P.= 2,3) e com o obstetra, foi de 8,1 consultas (D.P. $=3,4)($ Tabela 1$)$.

Quanto ao Diabetes Mellitus Gestacional, 25 mulheres (14\%) (IC 95\%, 9,3\% - 20,0\%) desenvolveram a doença ao longo da gestação; 34 $(24,5 \%)$ iniciaram a gestação com sobrepeso e 41 (29,5\%), com obesidade, considerando que só estavam disponíveis dados do estado nutricional pregresso de 139 participantes. Já em relação ao estado nutricional ao final da gestação, estavam disponíveis dados de 169 gestantes, dentre as quais $48(28,4 \%)$ apresentaram sobrepeso e $60(35,5 \%)$, obesidade (Figura 1).

$\mathrm{Na}$ análise da relação entre o estado nutricional pregresso e 0 desenvolvimento de DMG houve associação significativa $(p=0,001)$, sendo possível inferir que o estado nutricional pregresso esteve fortemente associado à ocorrência de diabetes nesta população (Tabela 2). Observou-se ainda que dentre as mulheres que desenvolveram DMG, sete $(20,6 \%)$ iniciaram a gestação com sobrepeso e 14 (34,1\%) apresentavam obesidade pregressa (Tabela 3). 
Tabela 1: Caracterização segundo perfil de consultas do pré-natal e informações sociodemográficas das gestantes atendidas no Ambulatório de Ginecologia e Obstetrícia em um Hospital Universitário, Niterói, RJ, Brasil, 2016.

\begin{tabular}{lccc}
\hline & Média & Desvio Padrão & N \\
\hline Idade materna (anos) & 27,7 & 7,2 & 178 \\
N $^{\circ}$ de consultas com obstetra & 8,1 & 3,4 & 178 \\
N $^{\circ}$ de consultas com nutricionista & 1,9 & 2,3 & 177 \\
\hline
\end{tabular}

Fonte: Dados da pesquisa realizada no Hospital Universitário de Niterói, RJ, março de 2016.

Figura 1: Casos de Diabetes Mellitus Gestacional de gestantes atendidas no Ambulatório de Ginecologia e Obstetrícia em um Hospital Universitário, Niterói, RJ. Brasil, 2016.

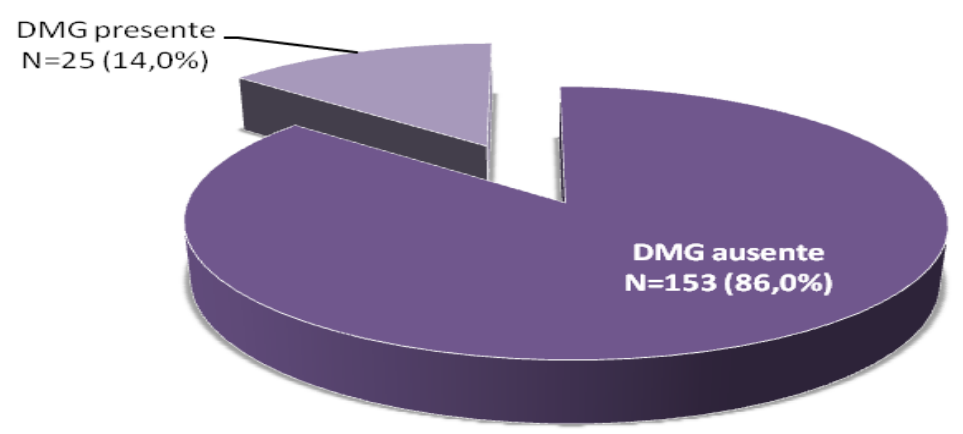

Fonte: Dados da pesquisa realizada no Hospital Universitário de Niterói, RJ, março de 2016. 
Tabela 2: Caracterização de gestantes atendidas em um Hospital Universitário, segundo diagnóstico do Diabetes Mellitus Gestacional e Classificação do Estado Nutricional, Niterói, RJ, Brasil, 2016.

\begin{tabular}{lcc}
\hline & $\mathbf{N}$ & $\%$ \\
\hline Diabetes Mellitus Gestacional & 178 & 100,0 \\
Não & 153 & 86,0 \\
Sim & 25 & 14,0 \\
& & \\
Classe de IMC - Pré gestacional & 139 & 100,0 \\
Baixo peso & 8 & 5,8 \\
Eutrofia & 56 & 40,3 \\
Sobrepeso & 34 & 24,5 \\
Obesidade & 41 & 29,5 \\
Classificação IMC gestacional - & 169 & 100,0 \\
ATALAH & & \\
Baixo peso & 16 & 9,5 \\
Adequado & 45 & 26,6 \\
Sobrepeso & 48 & 28,4 \\
Obesidade & 60 & 35,5 \\
\hline
\end{tabular}

Fonte: Dados da pesquisa realizada no Hospital Universitário de Niterói, RJ, março de 2016.

Tabela 3: Diabetes Mellitus Gestacional, segundo a classificação do Estado Nutricional Pré-gestacional de gestantes atendidas em um Hospital Universitário, Niterói, RJ, Brasil, 2016.

\begin{tabular}{|c|c|c|c|c|c|c|c|}
\hline & \multicolumn{4}{|c|}{ Diabetes Mellitus Gestacional } & \multirow{2}{*}{\multicolumn{2}{|c|}{ Total }} & \multirow{3}{*}{ P } \\
\hline & \multicolumn{2}{|c|}{ Não } & \multicolumn{2}{|c|}{ Sim } & & & \\
\hline & $\mathbf{N}$ & $\%$ & $\mathbf{N}$ & $\%$ & $\mathrm{~N}$ & $\%$ & \\
\hline $\begin{array}{l}\begin{array}{l}\text { Classificação de IMC Pré- } \\
\text { gestacional }\end{array} \\
\end{array}$ & 115 & 82,7 & 24 & 17,3 & 139 & 100 & 0,001 \\
\hline Baixo peso & 7 & 87,5 & 1 & 12,5 & 8 & 100 & \\
\hline Eutrofia & 54 & 96,4 & 2 & 3,6 & 56 & 100 & \\
\hline Sobrepeso & 27 & 79,4 & 7 & 20,6 & 34 & 100 & \\
\hline Obesidade & 27 & 65,9 & 14 & 34,1 & 41 & 100 & \\
\hline
\end{tabular}

Fonte: Dados da pesquisa realizada no Hospital Universitário de Niterói, RJ, março de 2016. 


\section{JOURNALOF \\ AND}

NURSING

\section{DISCUSSÃO}

O DMG é uma intercorrência muito comum em mulheres que apresentam histórico de sobrepeso pregresso ou que apresentam ganho ponderal inadequado durante a gestação. Ocorre em 1\% a $14 \%$ de todas

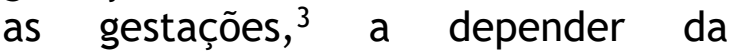
população estudada e do método de rastreio e diagnóstico. A alta taxa de DMG em um hospital de referência para gestantes de alto risco, constitui-se em problema de saúde pública e alerta para a necessidade de estratégias que possibilitem a prevenção e 0 tratamento desta enfermidade.

0 estudo sobre critérios para diagnóstico e prevalência de DMG em um hospital em Santa Catarina, Sul do Brasil, em uma coorte de 506 gestantes, evidenciou a prevalência de $14,4 \%{ }^{14}$ No entanto, um estudo realizado em uma maternidade pública na cidade de Maceió (Alagoas), Nordeste do Brasil, indicou que prevalência de DMG encontrada foi de 6,5\% dentre as 217 participantes daquele estudo. ${ }^{15}$

É certo que a prevalência pode variar de acordo com a população estudada, no entanto, os resultados apontados sugerem compatibilidade com o apresentado na literatura acima, ressaltando que este estudo foi conduzido em um hospital público de ensino, localizado no Sudeste do Brasil, região em que não foram encontrados estudos de prevalência.

O estado nutricional materno é apontado como fator de risco para o desenvolvimento do diabetes na gestação. A observação do estado
ISSN 2236 - 1987

nutricional pré-gestacional e o ganho de peso por idade gestacional, recomendam intervenção precoce com monitoramento constante do estado nutricional ao longo da gestação, já que a identificação de mudança de estado nutricional para sobrepeso ou obesidade no decorrer das semanas, sugere que esteja ocorrendo ganho de peso excessivo na gestação, havendo risco para a mulher e para o concepto em relação ao desenvolvimento de comorbidades associadas, tais como o diabetes e a hipertensão. ${ }^{14-15}$

O Ministério da Saúde recomenda o mínimo de seis consultas obstétricos ao longo do pré-natal. Nestas consultas, é esperado que as gestantes sejam monitoradas quanto ao estado nutricional e orientadas quanto aos hábitos alimentares compatíveis com suas necessidades. ${ }^{16}$ Um estudo em uma unidade de saúde de referência na Região Metropolitana de Porto Alegre, no Rio Grande do Sul, Brasil, 315 gestantes foram avaliadas quanto ao impacto de programas de orientação dietética sobre a velocidade de ganho de peso na gestação, tendo os autores concluído que as orientações alimentares foram efetivas na diminuição do ganho de peso naquelas que apresentavam previamente excesso de peso e, também, em reduzir intercorrências clínicas como o DMG, pré-eclâmpsia, baixo peso e prematuridade no grupo intervenção. ${ }^{17}$

Tanto o monitoramento do estado nutricional da gestante, quanto a promoção de saúde e novos hábitos de vida, com estratégias educacionais como rodas de conversas, laboratórios de práticas alimentares, além de contribuir para a melhor qualidade de 
vida da mulher, são condutas preventivas de baixo custo que podem ser efetivas e impactar positivamente na redução de novos casos da doença. ${ }^{18-25}$

\section{CONCLUSÃO}

O diabetes gestacional é uma comorbidade com associação ao estado nutricional materno e estudos que evidenciam o número de casos do diabetes gestacional e sua associação a fatores de risco, são importantes, principalmente no que tange à melhoria da assistência prestada e para a elaboração de novas estratégias de cuidado no ciclo gravídicopuerperal.

O DMG é uma enfermidade que pode desencadear o Diabetes tipo 2 e, portanto, é desejável que novos estudos sejam realizados para avaliar a associação entre DMG e fatores de risco nutricionais, considerando que a prevalência dessa doença pode variar de acordo com a população estudada, como evidenciado nos diferentes resultados de estudos encontrados em países da América Latina e entre regiões no Brasil.

Além disso, este estudo abrangeu o ano de 2013 num cenário de alta complexidade, e os resultados apresentados sugerem uma realidade que precisa ser modificada em função da alta prevalência da DMG. Ressaltase que novos estudos devem ser realizados no cenário nacional e internacional considerando que, com o avanço do status nutricional para sobrepeso e obesidade da população mundial, são necessárias novas
ISSN $2236-198$

evidências para
tratamento do DMG

\section{REFERÊNCIAS}

1. Sorbye LM, Skjaerven R, Klungsoyr K, Morken NH. Gestational diabetes mellitus and interpregnancy weight change: a populationbased cohort study. PLoS Med [Internet]. 2017 ago [acesso em 2018 jul 27]; 14(8):e1002367. Disponível em: https: //www.ncbi.nlm.nih.gov/pmc/a rticles/PMC5538633/

2. Ministério da Saúde (BR). Secretaria de Atenção à Saúde. Departamento de Ações Programáticas Estratégicas. Gestação de alto risco: manual técnico 5. ed. Brasília; 2010.

3. Diretrizes da Sociedade Brasileira de Diabetes: 2014-2015/Sociedade Brasileira de Diabetes; [organização José Egidio Paulo de Oliveira, Sérgio Vencio]. São Paulo: AC Farmacêutica, 2015.

4. Araújo MFM, Pessoa SMF, Damasceno MC, Zanetti ML. Diabetes gestacional na perspectiva de mulheres grávidas hospitalizadas. Rev bras enferm. [Internet]. 2013 jul/br[acesso em 2018 jul 27\};66(2):222-7. Disponível em: http://www.scielo.br/pdf/reben/v66 $\mathrm{n} 2 / 11 . \mathrm{pdf}$

5. Burbano-López RM, CastañoCastrillón JJ, González-Castellanos L, González-Henao HS, Quintero-Ospina $J D$, Revelo-Imbacuan $L$ de J, et al. Frecuencia de Diabetes Mellitus Gestacional y factores de riesgo en gestantes atendidas en clínicas de ASSBASALUD ESE, Manizales (Colombia) 2011-2012: estudio de corte 


\section{JOURNALOF \\ NURSING \\ AND HEALTH}

transversal. Rev colomb obstet ginecol [Internet]. $2014 \mathrm{mai} / \mathrm{dez}$ [acesso em 2018 jul 27];65(4):338-48. Disponível em:

http: / /www.scielo.org.co/pdf/rcog/v 65n4/v65n4a08.pdf

6. Benhalima K, Van Crombrugge $P$, Hanssens M, Devlieger R, Verhaeghe J, Mathieu C. Gestational diabetes: overview of the new consensus screening strategy and diagnostic criteria. Acta clin belg [Internet]. 2012 jul/ago[acesso em 2018 jul 27]; 67(4):255-61 Disponível em: https://www.tandfonline.com/doi/ab s/10.2143/ACB.67.4.2062669

7. Oteng-Ntim E, Mononen S, Sawicki O, Seed PT, Bick D, Poston L . Interpregnancy weight change and adverse pregnancy outcomes: a systematic review and mata-analysis. BMJ Open [Internet]. 2018 jun [acesso 2018 jul 12]; 8(6): e018778. Disponível em:

https://bmjopen.bmj.com/content/b mjopen/8/6/e018778. full.pdf

8. Schwartz N, Green MS, Yefet E, Nachum Z. Modifiable risk factors for gestational diabetes recurrence. Endocrine [Internet]. 2016 sep[acesso em 2018 apr 18];54(3)714-722. Disponível em: https://link.springer.com/article/10. 1007\%2Fs12020-016-1087-2

9. Reichelt AJ, Weinert LS, Mastella LS, Gnielka V, Campos MA, Hirakata VN, et al. Clinical characteristics of women with gestational diabetes - comparison of two cohorts enrolled 20 years apart in southern Brazil. Sao Paulo med j [Internet]. 2017 jul/ago [acesso em 2018 jul 27];135(4):376-82. Disponível
ISSN 2236 - 1987

em:

http:/ /www.scielo.br/pdf/spmj/v135 n4/1806-9460-spmj-1516-3180-20160332190317.pdf

10. Zajdenverg $L$, Negrato CA. Gestational diabetes mellitus and type 2 diabetes: same disease in a different moment of life? Maybe not. Arch. Endocrinol. Metab. [Internet]. 2017 mai/jun [acesso em 2018 jul 27];61(3):208-10. Disponível em: http:/ /www.scielo.br/pdf/aem/v61n3 10004-2730-aem-61-3-0208.pdf

11. Cnattingius $S$, Villamor E. Weight change between successive pregnancies and risks of stillbirth and infant mortality: a nationwide cohort study. Lancet [Internet]. $2016 \mathrm{dez} / \mathrm{fev}$ [acesso em 2018 jul 27]; 387(10018):558-65. Disponível em: https://www.thelancet.com/pdfs/jou rnals/lancet/PIIS0140-6736(15)009903.pdf

12. Opie RE, Neff M, Tierney AC. A behavioural nutrition intervention for obese pregnant women: effects on diet quality, weight gain and the incidence of gestational diabetes. Obstetrics \& Gynecology [Internet]. 2016 mai/ago [acesso em 2018 jul 27]; 56:364-73. Disponível em: https://obgyn.onlinelibrary.wiley.com /doi/epdf/10.1111/ajo.12474

13. World Health Organization (WHO). Physical status: the use and interpretation of anthropometry. Geneva: WHO; 1995.

14. Zapeline RM, Martinelli MT, João RM, Iser BPM. Critérios diagnósticos e prevalência de Diabetes Mellitus Gestacional em um hospital do sul de Santa Catarina. Revista da AMRIGS 


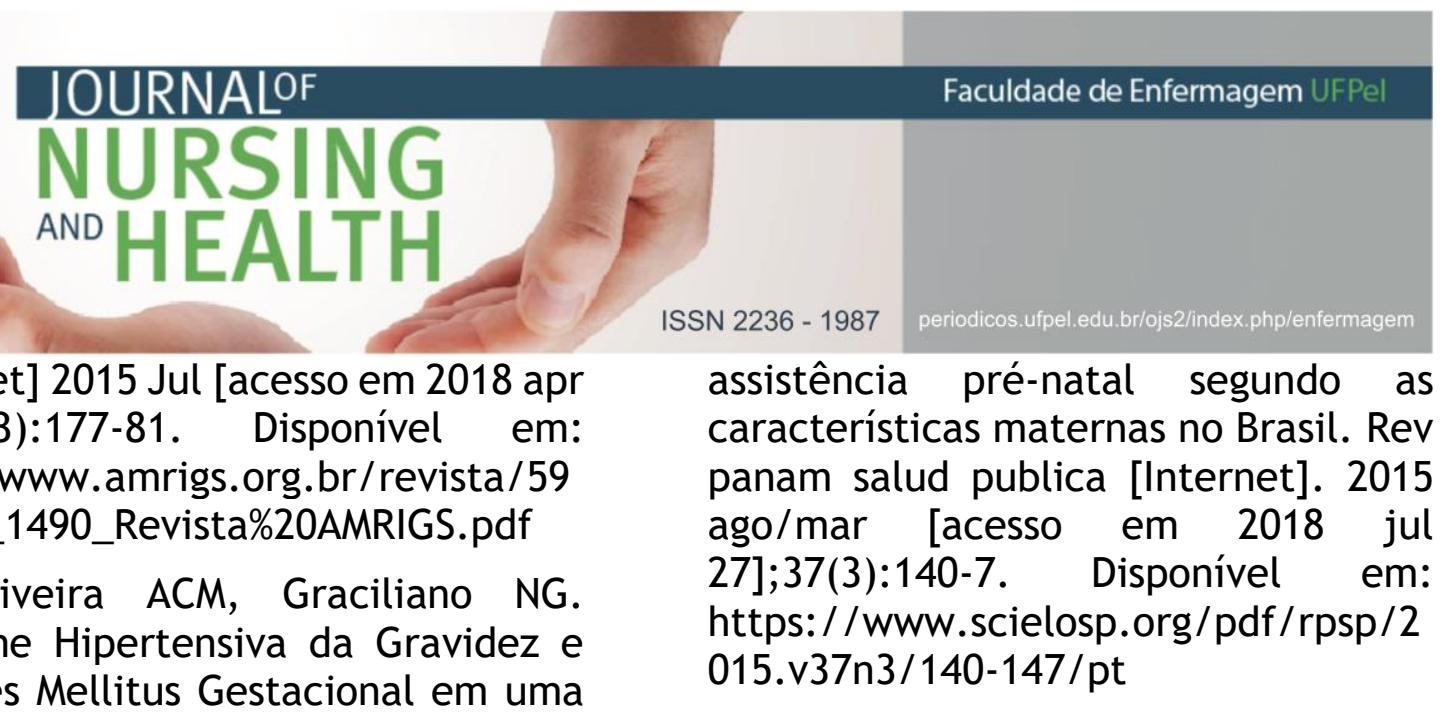

20. Neta FAV, Crisóstomo VL, Castro RCMB, Pesso SMF, Aragão MMS, Calou CGP Avaliação do perfil e dos cuidados no pré-natal de mulheres com diabetes mellitus gestacional. Rev rene (online) [Internet]. 2014 [acesso em 2018 jul 27];15(5):823-831. Disponível em: http: / / repositorio.ufc.br/bitstream/ri ufc/10657/1/2014_art_cgpcalou.pdf

16. Ministério da Saúde (BR). Pré-natal e Puerpério. Atenção qualificada e humanizada. Manual Técnico. Brasília: MS; 2006.

17. Vitolo MR, Bueno MSF, Gama CM. Impacto de um programa de orientação dietética sobre a velocidade de ganho de peso de gestantes atendidas em unidades de saúde. Rev bras ginecol obstet [Internet]. 2011 ago/dez [acesso em 2018 jul 27];33(1):13-9. Disponível em: http://www.scielo.br/pdf/rbgo/v33n 1/a02v33n1.pdf

18. MacDonald SC, Bodnar LM, Himes KP, Hutcheon JA. Patterns of gestational weight gain in eraly pregnancy and risk of gestational diabetes mellitus. Epidemiology [Internet]. 2017 may [acesso em 2018 jul 12];28(3):419-427. Disponível em: https://www.ncbi.nlm.nih.gov/pmc/a rticles/PMC5378599/pdf/nihms845432 .pdf

19. Domingues RMSM, Viellas EF, Dias $M A B$, Torres JÁ, Theme-Filha $M M$, Gama SGN, et al. Adequação da

21. Sato APS, Fujimori E. Estado Nutricional e ganho de peso de gestantes. Rev latino-am enferm [Internet]. $2012 \mathrm{mai} /$ jun [acesso em 2018 jul 27];20(3):1-7. Disponível em: http: / / www.scielo.br/pdf/rlae/v20n3 /pt_a06v20n3.pdf

22. Vitolo MR. Nutrição: da gestação ao envelhecimento. 2. ed. Rio de Janeiro: Rubio; 2015.

23. Ricart W, López J, Mozas J, Pericot A, Sancho MA, González N et al. Body mass index has a greater impact on pregnancy outcomes than gestational hyperglycemia. Diabetologia [Internet]. 2005 set/jul [acesso em 2018 jul 27];48(9):1736-42. Disponível em:

https://link.springer.com/article/10. 1007\%2Fs00125-005-1877-1

24. Schmalfuss JM, Prates LA, Azevedo $M$, Schneider $V$. Diabetes Melito Gestacional e as implicações para o cuidado de enfermagem no pré-natal. Cogitare enferm [Internet]. 2014 out/dez [acesso em 2018 jul 


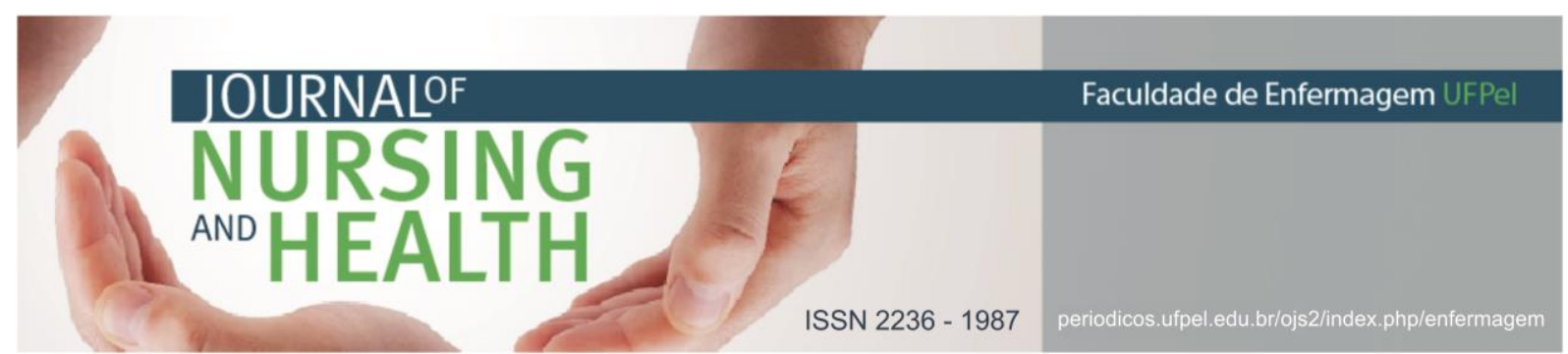

27];19(4):815-22. Disponível em: https://revistas.ufpr.br/cogitare/arti cle/view/36398/23961

25. Schmalfuss JM, Prates LA, Azevedo M, Schneider V. Diabetes Melito Gestacional e as implicações para o cuidado de enfermagem no pré-natal. Cogitare enferm [Internet]. 2014 out/dez [acesso em 2018 apr 27];19(4):815-22. Disponível em: https://revistas.ufpr.br/cogitare/arti cle/view/36398/23961

Data de submissão: 08/06/2017

Data de aceite: $11 / 07 / 18$

Data de publicação: 03/08/2018 